\title{
Eine Nacht im Notfalldienst
}

\author{
M. Hoppler
}

Das Telefon klingelt und Schwester Erika vom Ärztefon fragt freundlich, ob ich schon für den Dienst bereit wäre, denn heute Freitagabend sei richtig etwas los.

«Der erste Patient wohnt im Kreis 5, ein junger Mann, der sich nicht mehr bewegen kann. Bekannt sind chronische Rückenschmerzen, zurzeit aber so schlimm wie noch nie.».

Freitagabend 23.45 Uhr kommt man selbst in der Stadt Zürich mit dem Auto gut voran, und 10 Minuten später stehe ich an einem Küchentisch neben einem Patienten, welcher in einer unergonomischen Stellung dasteht. Er hat beide Hände auf den Tisch gestützt, und versucht möglichst keine Bewegung zu machen. Aufgrund seiner Schmerzen kann er nur noch mit dem Kopf nicken. Also übernimmt seine Frau für ihn das Sprechen. Nein, Allergien habe er keine und Medikamente nehme er auch nicht mehr ein, nur ab und zu Voltaren. Ja, Wasserlösen könne er noch, so geschehen vor 10 Minuten. Die körperliche Untersuchung zeigt über L3/L4 einen Rüttelschmerz und eine starke Druckdolenz, welche Herr S. die Schweissperlen auf die Stirn treibt. Neurologisch unauffällig, Motorik schmerzbedingt eingeschränkt. Er bekommt eine Spritze mit NSAR und Steroiden. Die Lagerung erkläre ich seiner Frau und am Montag wird er sich bei seinem Hausarzt melden. Reservemedikation mit Tramal und Voltaren. Frau S. bietet mir einen Espresso an. Da mir schon zwei neue Patienten telefonisch angemeldet wurden, lehne ich dankend ab.

Wieder im Auto geht es diesmal nach Schwamendingen.

In der Stadt sind nun die Nachtschwärmer unterwegs, von den Bars in die Clubs und umgekehrt, wodurch mein Fortkommen etwas gehemmt wird, einen Stau am Rosengarten umgehe ich elegant durch den Milchbucktunnel.

In einem städtischen Altersheim lerne ich eine nette Dame (1919) kennen, mit Fieber um $38,0^{\circ}$, welche sich einfach nicht «wohl» fühlt. Seit 1 Woche hütet sie nun schon das Bett. Sättigung 94\%, Atmung 24/min, kardial kompensiert, aber auskultatorisch ein Befund über dem linken Unterlappen. Der CRP-Schnelltest zeigt $>200$ sq. an. Ins Spital will Frau P. auf gar keinen Fall, aber mit den Antibiotika ist sie einverstanden. Am Montag verspricht sie mir, zum Hausarzt zu gehen oder mir zwischenzeitlich zu telefonieren, falls es ihr nicht besser geht. Ja, ihren Hausarzt, den kennt sie schon lange, als bei ihrem Ehemann vor 13 Jahren die Darmgeschichte begann, hat er die Betreuung übernommen. Kurz darauf verstarb er dann, ihr Mann. Dort auf dem Tisch steht sein Bild, daneben auf einer schönen weissen Spitzendecke, welche sie selber geklöppelt hat, das der Enkelin. Und ihre Schwester lebt jetzt auch in einem Altersheim, sie haben gerade noch miteinander telefoniert. 30 Minuten später und ziemlich gut über die weiteren Familienverhältnisse von Frau P. informiert, mache ich mich auf den Weg nach Altstetten.

Dort wartet ein 18jähriger Junge mit extremen Schmerzen im rechten Unterbauch. Seine Mutter öffnet die Türe. Luca ist auf das Sofa im Wohnzimmer gebettet. Dort hält sich auch seine kleinere Schwester auf, die sich gerade das PrettyWoman-Video anschaut.

Gegessen zum Frühstück hat er mit der Familie, am Mittag ein Pouletsandwich in der Schule und abends noch gar nichts, da die Schmerzen permanent zunahmen. Einmal erbrochen, einmal Durchfall. Die Schmerzen kommen und gehen. Ein bisschen dumpf, ein bisschen stechend, «es tut einfach weh!». Ich bitte die Familie, kurz draussen zu warten, Pretty Woman muss halt warten. Darmgeräusche lebhaft, keine Abwehrspannung, Druckdolenz über dem Nabel und Unterbauch rechts, kein Loslassschmerz, rektal nicht eindrücklich, kein Psoaszeichen. Fieber $38,0^{\circ}$ keine wesentliche Differenz. Wahrscheinlich ist es kein Blinddarm, aber ich schicke ihn trotzdem ins Spital, da mir sein Zustand einfach nicht gefällt.

Ein Telefon an die Sanität und ins Spital, dass ich einen «unklaren Bauch» zur Beurteilung schicke. Der Assistenzarzt ist zwar freundlich, aber nicht sehr begeistert. Ich bitte ihn, mir unbedingt einen Austrittsbericht $\mathrm{zu}$ senden. (Nachträglich stellt es sich als akute Blinddarmentzündung heraus.)

Auf dem Weg zum nächsten Patienten erledige ich noch zwei Telefonanrufe, eine Patientin, welche von ihrem Zahnarzt Ponstan verschrieben bekommen hat und nun die Dosierung nicht mehr weiss, und ein Patient, welcher nicht schlafen kann, aber keine Medikamente nehmen möchte. 
Die Strassen sind nun menschenleer, aber leider haben die Blitzlichter keine Nachtruhe und schon wieder habe ich ein neues in meiner Sammlung.

Kurze Zeit später stehe ich in der Wohnung eines 72jährigen Patienten mit bekannter Hypertonie, welcher seit 3 Tagen leichtes Fieber hat. Heute abend hat er sich früh ins Bett begeben, da er sich nicht wohl fühlte. Aufgewacht vor einer Stunde mit leichter Atemnot, welche er aber auf seine Erkältung zurückführte. Keine COPD bekannt.

Der Patient liegt im Bett, BD 140/80, P 100, auskultatorisch leise Herztöne, aber die Lunge brodelt über allen Lungenfeldern. Ich bitte ihn sich aufzusetzen. Nun sitzt er auf dem Bettrand und atmet schwer, Sättigung 72\%, ich gebe ihm Sauerstoff über Maske. Seine Frau erzählt mir, dass er aktuell versuche, etwas Gewicht zu reduzieren (BMI von 36). Sie holt die Medikamente aus dem Wandschrank: Aspirin cardio und Fluimucil, Panadol sowie ein Blutdruckmittel. Herr Z. beklagt sich über hypogastrische Schmerzen. Ich spritze $40 \mathrm{mg}$ Lasix iv. Als ich gerade die EKGElektroden anklebe, beginnt der Patient unruhig zu werden. Sättigung jetzt $82 \%$. Der Monitor zeigt SR an um 92/min, 30 Sekunden später Kammerflimmern und der Patient sackt nach hinten auf das Bett. Oh nein! Ich beginne sofort mit CPR, dazwischen Tel. 144 REA - bitte schnell - Hilfe! (an welcher Strasse sind wir schon wieder?), Defibrillator an, Laden 200J, Schock keine Reaktion, nochmals 300J, dann max. 360J, nach dem 3. Schock vent. Ersatzrhythmus mit 23 bpm, CPR, Sauerstoff umhängen, Guedel rein, oxigenieren, Pupillen weit, ich hätte gerne eine bessere Unterlage, kann den Pat. wegen seiner Lage und seines Gewichts aber nicht bewegen. Absaugen, der Rhythmus wird immer bradykarder, CPR, Sättigung zeigt nichts mehr an, ich versuche zu oxigenieren, und sauge nochmals $a b$, aber der Widerstand wird immer grösser. Dann Asystolie, in der Ferne hört man eine Sirene. Pupillen weit, wie lange bin ich schon dran? Es scheint eine Ewigkeit. Zum Glück kommt die Sanität. Endlich genug Hände. Wir machen weiter, aber ohne Erfolg, die Pupillen sind weit und reagieren nicht. Der Notarzt aus dem Spital erscheint, und wir entscheiden uns nach kurzer Diskussion, abzubrechen.

Die Sanitäter helfen noch aufzuräumen, das Zimmer gleicht einem Schlachtfeld. Als erstes spreche ich mit der Ehefrau, sie wartet in der Stube. Auf mein Drängen hin informiert sie ihre Tochter, welche in einer anderen Stadt lebt, ich spreche auch noch kurz am Telefon mit ihr. Ich fülle den Totenschein aus. Danach begleite ich sie zu ihrem Ehemann, um Abschied zu nehmen. Ich bleibe noch einen Moment bei ihr und organisiere auf ihren Wunsch hin den Abtransport des Verstorbenen.

Zurück beim Auto habe ich eine Parkbusse über Fr. 40.-, trotz des Schildes «Notfallarzt» und gültiger Vignette. Der diensthabende Wachmeister meint, dass er mit meinem Anruf gerechnet hätte. Er könne aber nicht wissen, ob ich bei Freunden zu Besuch sei oder ob ich im Dienst bin. Ich frage mich, wie prekär denn die Parkplatzsituation in dieser Stadt mittlerweile geworden ist.

$\mathrm{Zu}$ der nächsten Patientin fahre ich wie in Trance. Die REA beschäftigt mich noch weiter. Für die Ehefrau muss dies ja ein riesiger Schock gewesen sein. Hätte er doch nur nach dem ersten Schock einen anständigen Rhythmus gehabt, vielleicht hätte er dann ...

Frau G. lebt mit ihrem Ehemann und den zwei Kindern in einem Haus am Zürichberg. Ihr Ehemann im Bademantel öffnet die Türe und meint nur «wir haben sie früher erwartet».

Das Ehepaar war eingeladen bei Freunden zum Abendessen. Es gab zum Apéro Krabbenbrötchen, Lachs und Thunfischsushi, danach Lammfilet und Kartoffelgratin. Zum Dessert Mousse au Chocolat. Das Erbrechen setzte bei ihr 5 Stunden nach dem Hauptgang ein. Seither Erbrechen und starke Bauchkrämpfe, vor einer halben Stunde erstmalig Durchfall, kein Fieber. «Gut ich gebe Ihnen eine Spitze gegen das Erbrechen und etwas, um den Durchfall zu stoppen. Allergien haben Sie keine? Nein, aber seit etwa 2 Stunden eine leichte Sehstörung» - Kam der Thunfisch aus der Dose, oder die Krabben? Ist von den Freunden auch jemand krank? Auf mein Drängen hin werden die Gastgeber telefonisch geweckt und etwas verärgert erklären sie, dass der Thunfisch und die Krabben frisch gewesen und keine weiteren Krankheitsfälle bei den anderen Gästen bekannt seien. Ich lasse der Familie noch ein Rezept da und fahre in entgegengesetzter Richtung weiter. Inzwischen habe ich zwei weitere Patienten, die warten, und eine verstorbene Patientin im Altersheim. Ihr Abtransport sollte unbedingt noch vor dem Schichtwechsel organisiert werden, meint der zuständige Pfleger. (Das erledige ich dann auf dem Heimweg.)

Der nächste Patient kommt aus Somalia und ist hier nur zu Besuch. Die Haustüre mit der angegebenen Nummer gibt es nicht, und ich rufe die Natelnummer an, welche der Patient hinterlassen hat. In dieser Gegend sind die Häuser schlecht beleuchtet und die Hauseingänge nicht angeschrieben. Die Klingelknöpfe sind meist nur 
mit Initialen oder Zetteln beschriftet. Ich werde über einen Hinterhof und zwei Gänge, welche mit Velos, Schuhen und Containern verstopft sind, in ein kleines Zimmer gelotst.

Dort liegt auf einer Strohmatte ein etwa 20jähriger Mann. Betreut wird er von 3 Frauen in traditioneller Kleidung, welche neben ihm knien. Auf dem Sofa (das einzige Einrichtungsstück neben dem Fernseher) sitzen 3 Männer unterschiedlichen Alters. Niemand spricht meine Sprache und durch Gesten verstehe ich, dass ich warten soll. Ein Cousin des Patienten kommt, und wir unterhalten uns auf Französisch.

Der junge Mann hat Schwindel und fühlt sich nicht gut. Er ist seit 2 Tagen in der Schweiz. Die Reise hierhin sei ziemlich anstrengend gewesen. Kein Erbrechen, kein Durchfall. BD 130/80, P 105, Temp. 36,0․ Somatische Untersuchung bis auf kühle feuchte Haut unauffällig. Durch weitere Gesten erfahre ich vom Patienten, dass er heute etwas getrunken hat. Nein, keinen Alkohol, keine Drogen, aber es stellt sich heraus, dass es 3 Büchsen Red Bull waren. Er wird vermutlich die Nacht nicht schlafen, aber sonst geht es ihm gut. Ich erkläre ihm durch seinen Cousin, dass es nicht gefährlich ist, er aber in $\mathrm{Zu}$ kunft weniger Red Bull trinken soll.

Nur etwa $500 \mathrm{~m}$ weiter wartet mein nächster Patient auf mich, Herr J., 1920 geboren, welcher seit Jahren an Asthma leidet und heute abend einfach keine Ruhe findet. Seine Frau ist beunruhigt, weil er immer zu lange wartet, bis er den Arzt ruft. Er hat eine Sättigung von $82 \%$ und hat seine Arme fest auf den Stubentisch gepresst, eupnoisch ist er nicht gerade. Ich inhaliere zweimal mit ihm und gebe ihm einen Steroidstoss sowie Sauerstoff. Daraufhin verbessert sich sein AZ drastisch, und er kann wieder normal sprechen.

Er hat, wie er mir nun selber erzählt, auf die inhalativen Steroide verzichtet, da es ihm immer so gut gegangen ist. Ich erkläre ihm nochmals, dass es wichtig ist, bei der Therapie zu bleiben.

Nun geht es in den Kreis 7 zu einem 50jährigen Patienten mit Brustschmerzen. Er hat diese Schmerzen öfters, letztmalig vor einer Stunde, aber auch schon vor 2 und 4 Tagen. Als ich bei ihm ankomme, ist er fast schmerzfrei. Ein EKG zeigt leichte ST-Hebungen. Ich gebe Sauerstoff, Nitro und Aspirin, organisiere einen Transport ins Spital per Ambulanz. Der erste Jourarzt, dem ich telefoniere, hat keinen IPS-Platz mehr und verweist mich an ein anderes Spital, welches laut Liste einen Platz hat. Dieser ist aber auch schon vergeben, und das dritte Spital hat auch keinen Platz mehr. Bleibt nur noch das Universitätspital, der Jourarzt hat nur noch Platz im Notfall, übernimmt aber zum Glück den Patienten. Ich kann ihm noch Morphium verabreichen, da er unter den Nitraten nicht ganz schmerzfrei geworden ist. Als ich einen venösen Zugang legen will, ist die Ambulanz schon da und übernimmt das. Mit dem Blut aus dem venösen Zugang setze ich einen Troponin-Schnelltest an. Kurz einen Brief für das Spital geschrieben, und ab mit dem Patienten. Ich telefoniere nochmals mit dem Jourarzt und gebe ihm das positive Ergebnis durch.

Wieder im Auto melde ich mich bei meiner Zentrale ab, inzwischen ist es 7.15 Uhr und ich wünsche Schwester Erika noch einen kurzen Dienst. 